\title{
CORRESPONDENCE.
}

\section{ON A TABLE OF MORTALITY DEDUCED FROM THE NEW EXPERIENCE OBSERVATIONS, $\mathrm{H}^{\mathrm{MP}}$. \\ To the Editor of the Assurance Magazine.}

SIR,-Having recently constructed a Table of Mortality, based upon the Healthy Male and Female Observations published by the Institute of Actuaries, from which the number exposed to risk and the deaths during the first three years of Assurance-i.e., years 0, 1, and 2-have been eliminated, I beg to place the same at your disposal for insertion in the Journal of the Institute, should you consider the Table sufficiently interesting and useful for publication. It has been very carefully prepared, and has been graduated by the "New Method of Adjusting Mortality Tables," proposed and explained by $\mathrm{Mr}$. Woolhouse in the last volume of the Journal.

In explanation of my reason for excluding years of Assurance 0, 1, and 2 only, I may point out that the mortality amongst Male lives, which form 88 percent of the whole of my Table, during those three years taken together can be shown, by reference to $\mathrm{Mr}$. Sprague's exhaustive paper "On the rate of Mortality amongst Assured Lives as influenced by the Duration of the Assurance" (vol. xv., p. 338), to be 68.87 percent of the expectation by the 17 Offices' Experience, and 72.34 percent of the expectation by the New $\mathrm{H}^{\mathrm{M}}$ Experience itself, whereas the two succeeding years, 3 and 4, give $99 \cdot 13$ percent of actual to expected deaths by the 17 Offices' Table, and 102.84 percent by the Now Experience Table. The Experience Committee have excluded years of Assurance 0 to 4 from the $\mathrm{H}^{\mathrm{a}}$ Observations, and, I anderstand from Mr. Woolhouse's paper, 
recommend the resulting table "for the general purposes of valuations," although the actual deaths during the two years 3 and 4 thus thrown out are quite cent percent of the expected mortality. In this way 166,166 years of risk and 1188 deaths are rejected; and therefore I have thought it worth while to construct a Table, based upon the $\mathrm{H}^{\mathrm{MF}}$ Observations, and commencing at year of Assurance 3. Several eminent medical men express the opinion that the value of selection is practically lost after three years, and their opinion, though founded upon individual experience only, is confirmed by Mr. Sprague's investigations upon the subject, although there is no doubt that its effect is traceable for several years after. I venture however to suggest that the Table now produced may be assumed to indicate with considerable accuracy the mortality which may be expected to prevail amongst the assurers in a Life Office, such assurers consisting of male and female lives in fair average proportions, and from whom the effect of medical selection has passed away.

I have computed the probability of dying in a year at each age by the entire adjusted $\mathrm{H}^{\mathrm{MF}}$ Table given by Mr. Woolhouse on page 396 of the last volume of the Jour nal, and have placed them side by side with the corresponding probabilities deduced from the partial $\mathrm{H}^{\mathrm{MF}}$ Table to facilitate comparison. The diminished mortality at ages 10 to 18 by the partial experience, as compared with the total experience, is chiefly attributable to the small number of facts observed upon in both cases; but it is also due to the heavier mortality which prevailed at those ages in the two years immediately succeeding entry. It might therefore be advisable, in constructing monetary values, to disregard the probabilities of dying at ages 10 to 18 inclusive in favour of those obtained from the total experience. If this be done, the following would be the adjusted numbers-living and decrements at ages 10 to 24 , to be substituted for those in the Table.

\begin{tabular}{|c|c|c|c|c|c|c|c|c|}
\hline Age. & \multicolumn{1}{|c|}{$l_{x}$} & $d_{\boldsymbol{x}}$ & Age. & $l_{\boldsymbol{x}}$ & $d_{\boldsymbol{x}}$ & Age. & $l_{\boldsymbol{x}}$ & $d_{x}$ \\
\hline & & & & & & & & \\
\hline 10 & 100,410 & 455 & 15 & 98,427 & 389 & 20 & 95,947 & 727 \\
11 & 99,955 & 410 & 16 & 98,038 & 425 & 21 & 95,220 & 808 \\
12 & 99,545 & 380 & 17 & 97,613 & 477 & 22 & 94,412 & 834 \\
13 & 99,165 & 367 & 18 & 97,136 & 545 & 23 & 93,578 & 850 \\
14 & 98,798 & 371 & 19 & 96,591 & 644 & 24 & 92,728 & 846 \\
\hline
\end{tabular}

Taking however the two Tables as they stand, it will be noticed that the exclusion of the first three years of Assurance reveals an increased rate of mortality commencing at age 19 , and that such increase progresses rapidly until it reaches a maximum at age 24 , at which age the mortality is 32 percent more by the partial than by the total experience. The difference then begins to decrease with more or less regularity until the age of 80 , after which a change occurs, the mortality at ages 81 to 85 being slightly more by the total experience than by the partial experience, which fact is due to the few admissions at those ages and to the superior vitality of the female lives included. The mortality by the partial experience then increases until the end of the Table. The following Table shows very clearly the comparative influence of medical selection upon the mortality at different ages. 
$\mathrm{H}^{M F}$ (adjusted).- Number of Deaths compared.

\begin{tabular}{|c|c|c|c|c|c|}
\hline Ages. & $\begin{array}{c}\text { Total } \\
\text { Experience. }\end{array}$ & $\begin{array}{c}\text { Partial } \\
\text { Experience. }\end{array}$ & Ages. & $\begin{array}{c}\text { Total } \\
\text { Experience. }\end{array}$ & $\begin{array}{c}\text { Partial } \\
\text { Experience. }\end{array}$ \\
\hline $10-19$ & $100 \cdot 00$ & $93 \cdot 54$ & $60-69$ & $100 \cdot 00$ & $102 \cdot 89$ \\
$20-29$ & $100 \cdot 00$ & $124 \cdot 21$ & $70-79$ & $100 \cdot 00$ & $100 \cdot 72$ \\
$30-39$ & $100 \cdot 00$ & 11223 & $80-89$ & $100 \cdot 00$ & $100 \cdot 39$ \\
$40-49$ & $100 \cdot 00$ & $107 \cdot 09$ & $90-99$ & $100 \cdot 00$ & $103 \cdot 12$ \\
$50-59$ & $100 \cdot 00$ & $104 \cdot 15$ & & & \\
\hline
\end{tabular}

It will be observed that the adjusted partial experience Table presents a secondary maximum of mortality at the age of 24 , the probability of dying at that age being greater than at the ages immediately preceding and succeeding it. Mr. Sprague has pointed out, in his paper above referred to, that this peculiarity occurs at the age of 22 in the adjusted $\mathrm{H}^{\mathrm{M}} \mathrm{T}$ 'able, and that the same fact is noticeable at the same age in $\mathrm{Mr}$. Berridge's adjustment of the Peerage Table. If, however, we examine the complete unadjusted $\mathrm{H}^{\mathrm{M}}$ Table, as well as the partial unadjusted $\mathrm{H}^{\mathbf{M F}}$ Table, we shall find that this maximum of morfality occurs at age 23 . The increased mortality at this particular age is still more clearly defined in the $\mathrm{H}^{M}$ Table, from which the first five years of Assurance have been excluded; and it is worthy of note that the observations of the Mortality of the Government Male Life Annuitants by the late and present Actuaries to the National Debt show the same increase at that age, and further, that in the original Peerage Tables this maximum of mortality is indicated at age 23 in both the Male and Female observations. In reference to this point I would call attention to the following figures, and would remark that the facts upon which the percentages are computed are, I think, sufficiently numerous to command confidence in the results.

$\mathrm{H}^{\mathrm{M}}$ (unadjusted).-Mortality Percent.

\begin{tabular}{|c|c|c|c|}
\hline & Age 22 . & Age 23 & Age 24. \\
\hline 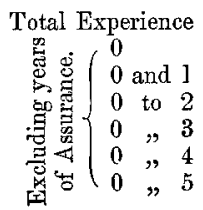 & $\begin{array}{r}.625 \\
.95] \\
.835 \\
.861 \\
1 \cdot 055 \\
1 \cdot 108 \\
1 \cdot 044\end{array}$ & $\begin{array}{r}\cdot 773 \\
.898 \\
1 \cdot 060 \\
1 \cdot 267 \\
1.660 \\
1 \cdot 705 \\
1 \cdot 728\end{array}$ & $\begin{array}{l}\cdot 686 \\
\cdot 779 \\
\cdot 764 \\
\cdot 765 \\
\cdot 643 \\
\cdot 460 \\
\cdot 560\end{array}$ \\
\hline
\end{tabular}

These figures are very remarkable, and, with one trifling exception which ascribes the maximum to age 22 , tend to confirm the opinion that it is at age 23 that the climax is reached. The increase of mortality at this age appears to be very decided and uniformly progressive in proportion to the length of time elapsed since selection, whereas at ages 22 and 24 the chance of dying fluctuates considerably when similarly examined in reference to the period when selection took place.

In conclusion, I give a few specimens of annuities and premiums computed at 4 percent interest, and based upon the adjusted $\mathrm{H}^{\mathrm{MF}}$ experience 
excluding the first three years of Assurance; and beg to add, that I shall be happy to furnish you with the complete 4 percent Table if desired.

\begin{tabular}{|c|c|c|c|}
\hline Age. & $\begin{array}{c}\text { Value of } \\
\text { Annuity of } £ l \text {. }\end{array}$ & $\begin{array}{c}\text { Value of } \\
\text { Reversion of } £ 1 .\end{array}$ & $\begin{array}{c}\text { Annual Premium } \\
\text { for Assurdnce } \\
\text { of } \mathscr{L} \text {. }\end{array}$ \\
\hline 10 & 197416 & 202244 & $\cdot 009751$ \\
\hline 15 & $18 \cdot 9855$ & $\cdot 231325$ & $\cdot 011575$ \\
\hline 20 & $18 \cdot 1930$ & 261806 & '013641 \\
\hline 25 & $17 \cdot 5818$ & 285314 & $\cdot 015355$ \\
\hline 30 & 16.8711 & $\cdot 312650$ & .017495 \\
\hline $3 \hat{b}$ & $16 \cdot 0145$ & $\cdot 345600$ & $\cdot 020812$ \\
\hline 40 & $15 \cdot 0099$ & $\cdot 384234$ & $\cdot 024000$ \\
\hline 45 & $13 \cdot 8265$ & -429750 & ·028985ั \\
\hline 50 & $12 \cdot 4985$ & $\cdot 480828$ & $\cdot 035621$ \\
\hline 55 & $11 \cdot 0439$ & $\cdot 536773$ & $\cdot 044567$ \\
\hline 60 & $9 \cdot 4777$ & -597010 & $\cdot 056978$ \\
\hline 65 & $7 \cdot 9032$ & $\cdot 657570$ & $\cdot 073858$ \\
\hline 70 & 6.3508 & $\cdot 717274$ & .097578 \\
\hline 75 & 49025 & $\cdot 772980$ & $\cdot 130958$ \\
\hline 80 & 37397 & $\cdot 817700$ & $\cdot 172522$ \\
\hline 85 & $2 \cdot 7026$ & 857592 & $\cdot 231619$ \\
\hline 90 & $1 \cdot 8861$ & •888996 & $\cdot 308027$ \\
\hline 95 & $1 \cdot 1431$ & $\cdot 917574$ & $\cdot 428156$ \\
\hline
\end{tabular}

I am, Sir,

Your obedient servant,

Cleveland House, WILFRED A. BOWSER. Lower Clapton, 17th October, 1870.

New Mortality Experience. $\mathrm{H}^{\mathrm{MF}}$, excluding the first Three Years of Assurance.

\begin{tabular}{|c|c|c|c|c|c|c|c|}
\hline \multirow{2}{*}{ Age. } & \multicolumn{2}{|c|}{ UNADJUSTED. } & \multicolumn{3}{|c|}{ ADJUATED. } & \multicolumn{2}{|c|}{$\begin{array}{l}\text { ProbabixtTy of Dying } \\
\text { IN A Year. }\end{array}$} \\
\hline & $\begin{array}{l}\text { Number- } \\
\text { living. }\end{array}$ & $\begin{array}{l}\text { Decre- } \\
\text { ment. }\end{array}$ & $\begin{array}{l}\text { Number- } \\
\text { living. }\end{array}$ & $\begin{array}{l}\text { Decre- } \\
\text { ment. }\end{array}$ & Expectation. & $\begin{array}{c}\text { Partial } \\
\text { Experience } \\
\text { Adjusted. }\end{array}$ & $\begin{array}{c}\text { Total } \\
\text { Experience } \\
\text { Adjusted. }\end{array}$ \\
\hline 10 & 100000 & 702 & 100000 & 354 & $48 \cdot 91$ & $\cdot 00354$ & .00442 \\
\hline 11 & 99298 & 0 & 99646 & 340 & $48 \cdot 08$ & $\cdot 00341$ & $\cdot 00409$ \\
\hline 12 & 99293 & 218 & 99306 & 337 & $47 \cdot 24$ & $\cdot 00339$ & .00388 \\
\hline 13 & 99080 & 190 & 98969 & 341 & $46 \cdot 40$ & .00344 & .00381 \\
\hline 14 & 98890 & 658 & 98628 & 358 & $45 \cdot 56$ & .00363 & .00385 \\
\hline 15 & 98232 & 602 & 98270 & 382 & $44 \cdot 72$ & .00389 & $\cdot 00404$ \\
\hline 16 & 97630 & 252 & 97888 & 416 & 4390 & .00425 & .00436 \\
\hline 17 & 97378 & 314 & 97472 & 461 & $43 \cdot 08$ & .00473 & $\cdot 00482$ \\
\hline 18 & 97064 & 356 & 97011 & 515 & 4228 & .00531 & $\cdot 00543$ \\
\hline 19 & 96708 & 831 & 96496 & 603 & $41 \cdot 50$ & .00625 & .00604 \\
\hline 20 & 95877 & 570 & 95893 & 701 & $40 \cdot 76$ & .00731 & .00649 \\
\hline 21 & 95307 & 854 & 95192 & 783 & 4006 & $\cdot 00822$ & 00679 \\
\hline 22 & 94453 & 820 & 94409 & 826 & $39 \cdot 39$ & .00875 & .00691 \\
\hline 23 & 93633 & 1113 & 93583 & 850 & 38.73 & .00908 & $\cdot 006.95$ \\
\hline
\end{tabular}


New Mortality Experience. $\quad \mathrm{H}^{\mathrm{MF}}, \& c .-($ continned.)

\begin{tabular}{|c|c|c|c|c|c|c|c|}
\hline \multirow{2}{*}{ Age. } & \multicolumn{2}{|c|}{$U_{N A D J C B T E D}$} & \multicolumn{3}{|c|}{ AdJOSTEd. } & \multicolumn{2}{|c|}{$\begin{array}{l}\text { YROBABILITY OF DYXNG } \\
\text { IN } A \text { YEAR. }\end{array}$} \\
\hline & $\begin{array}{l}\text { Number- } \\
\text { luving. }\end{array}$ & $\begin{array}{l}\text { Deere- } \\
\text { ment. }\end{array}$ & $\begin{array}{l}\text { Number- } \\
\text { living. }\end{array}$ & $\begin{array}{l}\text { Decre- } \\
\text { ment. }\end{array}$ & Expectation & $\begin{array}{c}\text { Partidal } \\
\text { Experience } \\
\text { Adjusted }\end{array}$ & $\begin{array}{c}\text { Total } \\
\text { Experience } \\
\text { Adjusted. }\end{array}$ \\
\hline 24 & 92520 & 783 & 92733 & 851 & $38 \cdot 08$ & $\cdot 00918$ & $\cdot 00695$ \\
\hline 25 & 91737 & 610 & 91882 & 840 & 3743 & .00914 & .00700 \\
\hline 26 & 91127 & 863 & 91042 & 802 & $36 \cdot 77$ & $\cdot 00881$ & $\cdot 00709$ \\
\hline 27 & 90264 & 826 & 90240 & 811 & $36 \cdot 10$ & .00899 & .00733 \\
\hline 28 & 89438 & 775 & 89429 & 830 & $35 \cdot 42$ & $\cdot 00928$ & .00758 \\
\hline 29 & 88663 & 898 & 88599 & 828 & $34 \cdot 75$ & $\cdot 00934$ & $\cdot 00783$ \\
\hline 30 & 87765 & 888 & 87771 & 830 & $34 \cdot 07$ & $\cdot 00946$ & $\cdot 00806$ \\
\hline 31 & 86877 & 761 & 86941 & 839 & $33 \cdot 39$ & $\cdot 00965$ & $\cdot 00823$ \\
\hline 32 & 86116 & 866 & 86102 & 830 & $32 \cdot 71$ & $\cdot 00964$ & $\cdot 00837$ \\
\hline 33 & 85250 & 812 & 85272 & 813 & $32 \cdot 02$ & $\cdot 00953$ & .00855 \\
\hline 34 & 84438 & 838 & 84459 & 825 & $31 \cdot 33$ & $\cdot 00977$ & $\cdot 00875$ \\
\hline 35 & 83600 & 740 & 83634 & 833 & 3063 & .00996 & $\cdot 00900$ \\
\hline 36 & 82860 & 870 & 82801 & 850 & 2993 & $\cdot 01026$ & $\cdot 00933$ \\
\hline 37 & 81990 & 885 & 81951 & 870 & $29 \cdot 24$ & .01062 & $\cdot 00967$ \\
\hline 38 & 81105 & 921 & 81081 & 893 & $28 \cdot 55$ & .01101 & .01000 \\
\hline 39 & 80184 & 935 & 80188 & 901 & 2786 & .01135 & .01027 \\
\hline 40 & 79249 & 898 & 79287 & 905 & $27 \cdot 17$ & .01141 & .01050 \\
\hline 41 & 78351 & 893 & 78382 & 907 & $26^{\circ} \cdot 48$ & .01157 & .01068 \\
\hline 42 & 77458 & 903 & 77475 & 911 & $25 \cdot 78$ & $\cdot 01176$ & .01091 \\
\hline 43 & 76555 & 899 & 76564 & 920 & 25.08 & $\cdot 01202$ & $\cdot 01127$ \\
\hline 44 & 75656 & 939 & 75644 & 938 & $24: 38$ & $\cdot 01240$ & .01173 \\
\hline 45 & 74717 & 981 & 74706 & 969 & 2368 & .01297 & .01232 \\
\hline 46 & 73736 & 979 & 73737 & 1007 & $22 \cdot 99$ & $\cdot 01366$ & .01301 \\
\hline 47 & 72757 & 1084 & 72730 & 1050 & $22 \cdot 30$ & $\cdot 01444$ & .01372 \\
\hline 48 & 71673 & 1061 & 71680 & 1087 & $2 \mathrm{l} \cdot 62$ & .01516 & .01442 \\
\hline 49 & 70612 & 1112 & 70593 & 1128 & $20 \cdot 94$ & .01598 & 01511 \\
\hline 50 & 69500 & 1224 & 69465 & 1163 & 2027 & $\cdot 01674$ & $\cdot 01577$ \\
\hline 51 & 68276 & 1218 & 68302 & 1189 & $19 \cdot 61$ & $\cdot 01741$ & $\cdot 01651$ \\
\hline 52 & 67058 & 1241 & 67113 & 1227 & $19 \cdot 38$ & $\cdot 01828$ & $\cdot 01732$ \\
\hline 53 & 65817 & 1142 & 65886 & 1268 & $18 \cdot 29$ & $\cdot 01924$ & 01831 \\
\hline 54 & 64675 & 1265 & $646 \mathrm{I} 8$ & 1314 & $17 \cdot 64$ & $\cdot 02033$ & .01945 \\
\hline 55 & 63410 & 1483 & 63304 & 1362 & 16.99 & $\cdot 02151$ & $\cdot 02065$ \\
\hline 56 & 61927 & 1457 & 61942 & 1413 & $16: 36$ & $\cdot 02281$ & $\cdot 02196$ \\
\hline 57 & 60470 & 1436 & 60529 & 1465 & $15 \cdot 72$ & $\cdot 02420$ & $\cdot 02336$ \\
\hline 58 & 59034 & 1540 & 59064 & 1523 & $15 \cdot 10$ & .02578 & $\cdot 02489$ \\
\hline 59 & 57494 & 1449 & 57541 & 1583 & $14 \cdot 49$ & $\cdot 02751$ & $\cdot 02669$ \\
\hline 60 & 56045 & 1750 & 55958 & 1661 & 1388 & .02968 & .02873 \\
\hline 61 & 54295 & 1693 & 54297 & 1737 & $13 \cdot 29$ & $\cdot 03199$ & .03104 \\
\hline 62 & 52502 & 1841 & 52560 & 1824 & 12.71 & .03470 & .03366 \\
\hline 63 & 50761 & 1952 & 50736 & 1911 & $12 \cdot 15$ & $\cdot 03766$ & $\cdot 03647$ \\
\hline 64 & 48809 & 1949 & 48825 & 1992 & $11 \cdot 61$ & $\cdot 04080$ & $\cdot 03937$ \\
\hline 65 & 46860 & 2094 & 46833 & 2047 & $11 \cdot 08$ & $\cdot 04371$ & .04233 \\
\hline 66 & 44766 & 2065 & 44786 & 2100 & $10 \cdot 57$ & $\cdot 04689$ & $\cdot 04543$ \\
\hline 67 & 42701 & 2124 & 42686 & 2137 & 30.06 & .05006 & $\cdot 04866$ \\
\hline 68 & 40577 & 2257 & 40549 & 2157 & $9 \cdot 566$ & .05319 & $\cdot 05204$ \\
\hline 69 & 38320 & 2304 & 38392 & 2189 & $9 \cdot 076$ & .05702 & $\cdot 05599$ \\
\hline 70 & 36016 & 2003 & 36203 & 2229 & 8.594 & $\cdot 06157$ & .06095 \\
\hline 71 & 34013 & 2087 & 33974 & 2281 & $8 \cdot 125$ & $\cdot 06714$ & $\cdot 06686$ \\
\hline 72 & 31926 & 2466 & 31693 & 2354 & $7 \cdot 674$ & $\cdot 07427$ & .07368 \\
\hline 73 & 29470 & 2393 & 29339 & 2404 & $7 \cdot 250$ & .08194 & $\cdot 08154$ \\
\hline 74 & 27077 & 2802 & 26935 & 2438 & 6.852 & .09051 & $\cdot 09004$ \\
\hline 75 & 24275 & 2222 & 24497 & 2404 & $6 \cdot 484$ & $\cdot 09813$ & $\cdot 09799$ \\
\hline 76 & 22053 & 2366 & 22093 & 2365 & $6 \cdot 135$ & $\cdot 10705$ & $\cdot 10581$ \\
\hline
\end{tabular}


New Mortality Experience. $\quad \mathrm{H}^{\mathrm{MF}}, \& c .-$ (continued).

\begin{tabular}{|c|c|c|c|c|c|c|c|}
\hline \multirow{2}{*}{ Age. } & \multicolumn{2}{|c|}{ UNADJDSTED. } & \multicolumn{3}{|c|}{ ADJUSTED. } & \multicolumn{2}{|c|}{$\begin{array}{l}\text { ProbabintTY of DYing } \\
\text { IN A YEA }\end{array}$} \\
\hline & $\begin{array}{l}\text { Number- } \\
\text { living. }\end{array}$ & $\begin{array}{l}\text { Decre- } \\
\text { ment. }\end{array}$ & $\begin{array}{l}\text { Number- } \\
\text { living. }\end{array}$ & $\begin{array}{l}\text { Decre- } \\
\text { ment. }\end{array}$ & Expectation & $\begin{array}{c}\text { Partial } \\
\text { Experience } \\
\text { Adjusted. }\end{array}$ & $\begin{array}{c}\text { Total } \\
\text { Experience } \\
\text { Adjusted. }\end{array}$ \\
\hline 77 & 19687 & 2113 & 19728 & 2257 & $5 \cdot 811$ & $\cdot 11441$ & •11322 \\
\hline 78 & 17574 & 2302 & 17471 & 2131 & $5 \cdot 497$ & $\cdot 12197$ & $\cdot 12110$ \\
\hline 79 & 15272 & 2017 & 15340 & 2001 & $5 \cdot 191$ & $\cdot 13044$ & $\cdot 12938$ \\
\hline 80 & 13255 & 1776 & 13339 & 1863 & 4.895 & $\cdot 13966$ & $\cdot 13868$ \\
\hline 81 & 11479 & 1762 & 11476 & 1710 & $4 \cdot 609$ & $\cdot 14901$ & $\cdot 14907$ \\
\hline 82 & 9717 & 1432 & 9766 & 1568 & $4 \cdot 328$ & $\cdot 16055$ & $\cdot 16068$ \\
\hline 83 & 8285 & 1536 & 8198 & 1426 & $4 \cdot 060$ & $\cdot 17394$ & $\cdot 17426$ \\
\hline 84 & 6749 & 1214 & 6772 & 1270 & 3.810 & $\cdot 18753$ & $\cdot 18857$ \\
\hline 85 & 5535 & 1211 & 5502 & 1115 & 3574 & $\cdot 20265$ & -20267 \\
\hline 86 & 4324 & 952 & 4387 & 955 & 3355 & $\cdot 21768$ & $\cdot 21732$ \\
\hline 87 & $\mathbf{3 3 7 2}$ & 751 & 3432 & 805 & $3 \cdot 150$ & $\cdot 23455$ & $\cdot 23248$ \\
\hline 88 & 2621 & 691 & 2627 & 651 & $2 \cdot 962$ & $\cdot 24781$ & $2458 \mathrm{I}$ \\
\hline 89 & 1930 & 454 & 1976 & 520 & $2 \cdot 773$ & 26316 & $\cdot 25923$ \\
\hline 90 & 1476 & 394 & 1456 & 411 & 2.585 & $\cdot 28228$ & 27778 \\
\hline 91 & 1082 & 309 & 1045 & 319 & $2 \cdot 405$ & $\cdot 30526$ & $\cdot 29708$ \\
\hline 92 & 773 & 304 & 726 & 233 & $2 \cdot 242$ & $\cdot 32093$ & 31069 \\
\hline 93 & 469 & 235 & 493 & 168 & $2 \cdot 066$ & $\cdot 34077$ & $\cdot 33029$ \\
\hline 94 & 234 & 0 & 325 & 124 & $1 \cdot 875$ & $\cdot 38154$ & $\cdot 35694$ \\
\hline 95 & 234 & 26 & 201 & 79 & $1 \cdot 724$ & $\cdot 38806$ & $\cdot 36441$ \\
\hline 96 & 208 & 130 & 122 & 48 & $1 \cdot 516$ & $\cdot 39837$ & $\cdot 37334$ \\
\hline 97 & 78 & 39 & 74 & 36 & $1 \cdot 207$ & $\cdot 48648$ & $\cdot 46809$ \\
\hline 98 & 39 & 0 & 38 & 26 & .815 & $\cdot 68420$ & $\cdot 65999$ \\
\hline 99 & 0 & 0 & 12 & 12 & $\cdot 500$ & $1 \cdot 00000$ & $1 \cdot 00000$ \\
\hline 100 & 0 & 0 & 0 & 0 & & & \\
\hline
\end{tabular}

\section{ON HERR LAZARUS'S PAPER ON THE THEORY OF PROBABILITIES.}

\section{To the Editor of the Journal of the Institute of Actuaries.}

SiR,--In the July number of the Journal you inserted a letter from me, having for its object the elucidation of a passage in Herr Lazarus's paper "On some problems in the Theory of Probabilities." I have since received a very courteous communication from Herr Lazarus in reference to the subject of my letter; and I beg to send you the substance of that communication out of fairness to Herr Lazarns, at the same time feeling confident that it will greatly interest some of your readers.

He says, in explanation of the passage upon which my remarks were based, "The simplest way to find the sum $\Omega_{0}+\Omega_{1}+\Omega_{2}$ would be to extend " one of the equations (28) or (29), so as to include $\Omega_{0}$. I think it is self" evident from (28) that

$$
\text { " } \Omega_{0}+\Omega_{1}=\frac{\int_{0}^{p} x^{m-1}(1-x)^{n} d x}{\int_{0}^{1} x^{m-1}(1-x)^{n} d x}-\frac{\int_{0}^{p} x^{m+z}(1-x)^{n-z-1} d x}{\int_{0}^{1} x^{m+z}(1-x)^{n-z-1} d x}
$$

